This is a repository copy of Logic-Based Traffic Flow Control for Ramp Metering and Variable Speed Limits--Part 2: Simulation and Comparisons, in the Depósito de Investigación de la Universidad de Sevilla

Version: Author Accepted Version

Citation: Jose Ramon D. Frejo, Bart De Schutter, Logic-Based Traffic Flow Control for Ramp Metering and Variable Speed Limits--Part 2: Simulation and Comparisons, IEEE Transactions on Intelligent Transportation Systems, pp: 1 - 11, Feb 2020. 10.1109/tits.2020.2973732

To cite this publication, please use the final published version (if applicable). Please check the document version above.

Copyright: Other than for strictly personal use, it is not permitted to download, forward or distribute the text or part of it, without the consent of the author(s) and/or copyright holder(s), unless the work is under an open content license such as Creative Commons.

Takedown policy: Please contact us (idus@us.es) and provide details if you believe this document breaches copyrights. We will remove access to the work immediately and investigate your claim 
This is a repository copy of Logic-Based Traffic Flow Control for Ramp Metering and Variable Speed Limits-Part 2: Simulation and Comparison in the Depósito de Investigación de la Universidad de Sevilla

Version: Author Accepted Version

Citation: J. R. D. Frejo and B. De Schutter, "Logic-Based Traffic Flow Control for Ramp Metering and Variable Speed Limits-Part 2: Simulation and Comparison," in IEEE Transactions on Intelligent Transportation Systems, vol. 22, no. 5, pp. 2658-2668, 2021.

DOI: 10.1109/TITS.2020.2973732.

Link: https://ieeexplore.ieee.org/document/9007042

To cite this publication, please use the final published version (if applicable). Please check the document version above.

(c) 2021 IEEE. Personal use of this material is permitted. Permission from IEEE must be obtained for all other uses, in any current or future media, including reprinting/republishing this material for advertising or promotional purposes, creating new collective works, for resale or redistribution to servers or lists, or reuse of any copyrighted component of this work in other works

Takedown policy: Please contact us (idus@us.es) and provide details if you believe this document breaches copyrights. We will remove access to the work immediately and investigate your claim 


\title{
Logic-based traffic flow control for ramp metering and variable speed limits (Part 2: Simulation and comparison)
}

\author{
José Ramón D. Frejo, and Bart De Schutter, Fellow, IEEE
}

\begin{abstract}
This paper simulates, analyzes, and compares, for two case studies (one synthetic freeway and one real-life freeway), the behavior of Logic-Based Traffic Flow Control (LB-TFC), an integrated control strategy for Ramp Metering (RM) installations and Variable Speed Limits (VSLs) that was proposed and derivated in the first part of the work ('Part 1: Controller').

For the first case study, which was presented in the first part of the work, the control performance of LB-TFC is compared with the ones obtained with the optimal solution and with the Mainstream Traffic Flow Control (MTFC) + PI-ALINEA algorithm. Moreover, the robustness of the considered controllers is analyzed for this case study concluding that LB-TFC is quite robust, specially when comparing with MTFC + PI-ALINEA.

For the second study (a stretch of the ring-road freeway SE30 in Seville, Spain), data from 10 different days have been used in order to simulate the performance of the considered controllers using real data for the afternoon peak period. In order to properly deal with a bottleneck with a dynamically changing number of lanes, the equations used for MTFC + PI-ALINEA have been slightly modified for the second case study.

For both case studies, LB-TFC provides a robust performance that, in most cases, is close to the optimal one and that improves the reduction in the Total Time Spent (TTS) obtained with MTFC + PI-ALINEA. Moreover, this paper studies the tuning of the control parameters and the advantages and disadvantages of LBTFC.
\end{abstract}

Index Terms-Ramp Metering, Variable Speed Limits (VSLs), Feed-forward control, Freeway traffic control, logic-based control.

\section{INTRODUCTION}

This work is divided in two parts, each one presented in a separate paper. In 'Part 1: Controller' [1], the control structure and the equations of LB-TFC have been presented and derived. In this paper ('Part 2: Simulation and Comparison'), LB-TFC is simulated, analyzed, and compared for two freeways (one synthetic network and one stretch of the ring-road freeway SE-30 in Seville, Spain).

The main contribution of this paper is to show by simulation that LB-TFC provides a robust behavior with a performance that is close to the optimal one. Other contributions of this paper are the comparative study of the robustness of both LBTFC and MTFC + PI-ALINEA, the modification of MTFC

J. R. D. Frejo is with the Dept. de Ingeniería de Sistemas y Automática, University of Seville, Spain. B. De Schutter is with the Delft Center for Systems and Control, Delft University of Technology, The Netherlands. E-mails: jdominguez3@us.es, B.DeSchutter@tudelft.nl

This research was supported by the European Union's Horizon 2020 research and innovation programee under the Marie Skłodowska-Curie grant agreement No 702579 and under the ERC Advanced Grant agreement No 789051.
+ PI-ALINEA in order to deal with a bottleneck with a dynamically changing number of lanes, and (to the best of the authors' knowledge) the first study that uses VSLs and RMs in order to alleviate the congestion created on a bottleneck with a reversible lane.

This paper is organized as follows: Firstly, the comparative analysis of results for the synthetic freeway stretch is presented, including a numerical and graphical comparison in Section II and an analysis of the robustness of the considered controllers in Section III. Subsequently, in Section IV, LBTFC is tested and compared using a stretch of the SE-30 freeway in Seville, Spain. Section V performs a simulationbased analysis of the effects of the tuning parameters on the response of LB-TFC. Section VI studies the advantages and disadvantages of LB-TFC with respect to other integrated controllers for RM installations and VSLs. Finally, the main conclusions are drawn in Section VII.

\section{Controller COMPARISON FOR CASE STUdy I}

In this section, the behavior and performance of LB-TFC is compared for Case Study I (a $12 \mathrm{~km}$ long synthetic freeway strech) with the Mainstream Traffic Flow Control (MTFC) + PI-ALINEA algorithm and with the optimal solution. The details about the case study, the considered scenarios, and the simulation results for LB-TFC are presented in Section IV of the first part of this work [1].

\section{A. Simulated controllers}

1) MTFC+PI-ALINEA: The integrated controller MTFC + PI-ALINEA [2] is probably the most well-known easy-toimplement control architecture for VSLs and RMs.

In most previous applications, MTFC has been applied for freeways where the on-ramp is placed downstream of the VSLs. However, another realistic situation is to consider that the VSLs are located downstream of the RM installation. In this case, assuming that the RM installation is going to be used before the VSLs, an equivalent control structure can be applied:

- The controller operates the RM installation using the control structure of PI-ALINEA until the RM installation is not able to resolve congestion.

- If the RM installation is not able to resolve congestion (i.e. if the queue is already saturated), the VSLs are operated as MTFC but computing the desired mainstream 
TABLE I

OPTIMAL CONTROL PARAMETERS FOR MTFC + PI-ALINEA OPTIMIZED FOR SCENARIO $i$

\begin{tabular}{cccccccccccc} 
Scenario & 1 & 2 & 3 & 4 & 5 & 6 & 7 & 8 & 9 & 10 & All \\
\hline $\bar{K}_{I}^{i}(\mathrm{~km} \cdot \mathrm{lane} / \mathrm{h})$ & 0.0124 & 8.5644 & 0.4553 & 1.1414 & 0.5092 & 0.1512 & 1.9601 & 7.0374 & 4.4150 & 5.6058 & 0.1083 \\
\hline $\bar{K}_{P}^{i}(\mathrm{~km} \cdot \mathrm{lane} / \mathrm{h})$ & 40.172 & 245.99 & 61.624 & 81.407 & 89.338 & 84.175 & 115.92 & 333.52 & 106.83 & 123.89 & 54.811 \\
\hline$K_{I}^{i}(\mathrm{~h} \cdot$ lane/veh$)$ & 0.00157 & 0.00174 & 0.00106 & 0.00028 & 0.00002 & 0.00002 & 0.00158 & 0.00008 & 0.00183 & 0.00054 & 0.00110 \\
\hline$K_{I}^{\prime}(\mathrm{km} / \mathrm{h})$ & 0.5309 & 6.5074 & 0.1826 & 6.6038 & 5.7214 & 0.1560 & 6.8944 & 4.3228 & 7.4539 & 8.7253 & 0.1115 \\
\hline$K_{P}^{\prime}(\mathrm{km} / \mathrm{h})$ & 134.36 & 47.551 & 109.69 & 83.928 & 73.542 & 60.952 & 171.517 & 53.303 & 55.433 & 48.209 & 83.062 \\
\hline
\end{tabular}

flow $\left(\hat{q}_{\mathrm{c}}\right)$ by subtracting the queue-management ordered inflow $\left(q_{\mathrm{r}}\right)$ to the reference flow $\left(\hat{q}_{\mathrm{t}}\right)$ as explained in [2].

It should be also noted that the operating gains of the primary controller are scheduled based on the split decision and that, if desired, the controller may be modified in order to apply the VSLs before the application of the RM.

2) Optimal control: The optimal solution for each scenario is computed in order to obtain an estimate of the highest Total Time Spent (TTS) reduction that can be achieved for each scenario. More concretely, the optimal solution between time steps $k_{\mathrm{o}}$ and $k_{\mathrm{e}}$ for a given demand can be found by solving the optimization problem with the following cost function :

$$
\begin{aligned}
& J=\sum_{k=k_{\mathrm{o}}+1}^{k_{\mathrm{e}}}\left[T\left(\sum_{i \in \mathrm{O}} w_{i}(k)+\sum_{i \in I} \rho_{i}(k) L_{i} \lambda_{i}\right)+\right. \\
& \psi\left(\max \left(w_{4}(k)-\bar{w}, 0\right)\right)^{2}+ \\
& \epsilon \sum_{i \in \mathrm{R}}\left(\mathrm{RM}_{i}(k)-\mathrm{RM}_{i-1}(k-1)\right)^{2}+ \\
& \Omega \sum_{i \in \mathrm{V}}\left(\frac{\mathrm{VSL}_{i}(k)-\mathrm{VSL}_{i-1}(k-1)}{v_{\mathrm{f}}}\right)^{2}
\end{aligned}
$$

where $\psi$ and $\epsilon$ are tuning parameters, $O$ is the set of all the origins (i.e. the on-ramp and the mainline origin), $R$ is the set of all the RM installations, $V$ is the set of all the VSLs, and $I$ is the set of all the segments. For this case study, $\psi$ and $\Omega$ have been set equal to 1 and $\epsilon$ has been set equal to 5 . If lower values of $\epsilon$ and/or $\Omega$ are used, the TTS is slightly reduced, but in general higher oscillations may appear in the control signals.

Cost function (1) contains one term for the TTS, another term that limits (using a soft constraint in order to make the optimization faster) the maximum value of the queues, and a third term penalizing the ramp metering rate and speed limits variations

The computation of the optimal RM and VSL solution has been analyzed in many previous references either using a rolling prediction horizon [3], [4] or optimizing over the entire simulation horizon [5]. In this work, we use the second choice because the computation load is not a key factor since the optimal solution is only used in order to analyze the performance of LB-TFC and MTFC + PI-ALINEA (the optimal solution provides an estimate of the best performance that can be achieved). The necessary optimizations have been computed continuously using the gradient-based optimization algorithms RPROP [5], [6] and Sequential Quadratic Programming (SQP)
[7]. It has to be pointed out that, in general, it is necessary to run the optimization algorithms many times (with different initial points) in order to avoid ending up in local minima (because the problem is highly non-convex). More concretely, 15 initial points have been used for each computation of the optimal solution, 100 initial points for the computation of the optimal values of $\bar{C}_{\mathrm{B}}^{i}, \underline{C}_{\mathrm{B}}^{i}$ for each scenario, and 100 initial points (pre-selected, based on the value of their cost function, from a grid of 100000 points in the feasible region) for the computation of $\bar{K}_{I}^{i}, \bar{K}_{P}^{i}, K_{I}^{i}, K_{I}^{\prime}$, and $K_{P}^{\prime} i$ for each scenario.

\section{B. Numerical results}

In this section, LB-TFC, MTFC + PI-ALINEA, and the optimal solution are tested and numerically compared for the 10 considered scenarios.

The values of the controller parameters used for the simulation of LB-TFC and MTFC + PI-ALINEA for each scenario $i\left(\bar{C}_{\mathrm{B}}^{i}, \underline{C}_{\mathrm{B}}^{i}, \bar{K}_{I}^{i}, \bar{K}_{P}^{i}, K_{I}^{i}, K_{I}^{\prime}\right.$, and $\left.K_{P}^{\prime}\right)$ have been found by minimizing the cost function (1) for the given scenario $i$. The parameters of LB-VSL are included in Table III of the first part of this work [1] and the parameters of MTFC + PIALINEA, which have been expressed with the same notation and units as in [2], can be seen in Table I.

The obtained numerical results are shown in Table II. Analyzing the results, it can be seen that the highest difference between the TTS reduction of LB-TFC and the optimal TTS reduction occurs for Scenario 5 (with a TTS reduction of $29.8 \%$ for the optimal case and $27.6 \%$ for the proposed controller). Therefore, it can be said that the proposed controller is able to approach the optimal performance for the entire set

\begin{tabular}{|c|c|c|c|c|}
\hline Scen. & $\begin{array}{l}\text { No } \\
\text { Control }\end{array}$ & Optimal & $\begin{array}{c}\text { MTFC + } \\
\text { PI-ALINEA }\end{array}$ & LB-TFC \\
\hline 1 & 2860.7 & $1455.2(-49.1 \%)$ & $1472.8(-48.5 \%)$ & $1455.7(-49.1 \%)$ \\
\hline 2 & 2860.7 & $1676.4(-41.4 \%)$ & $1759.4(-38.5 \%)$ & $1710.0(-40.2 \%)$ \\
\hline 3 & 3820.1 & $2390.3(-37.4 \%)$ & $2501.1(-34.5 \%)$ & $2458.6(-35.6 \%)$ \\
\hline 4 & 3820.1 & $2920.4(-23.6 \%)$ & $2959.7(-22.5 \%)$ & $3001.4(-21.4 \%)$ \\
\hline 5 & 3007.2 & $2111.5(-29.8 \%)$ & $2154.2(-28.4 \%)$ & $2175.7(-27.6 \%)$ \\
\hline 6 & 3007.2 & $2479.9(-17.5 \%)$ & $2511.2(-16.5 \%)$ & $2498.8(-16.9 \%)$ \\
\hline 7 & 2464.8 & $1794.1(-27.2 \%)$ & $1844.9(-25.1 \%)$ & $1825.9(-25.9 \%)$ \\
\hline 8 & 2464.8 & $2024.9(-17.8 \%)$ & $2049.8(-16.8 \%)$ & $2049.4(-17.0 \%)$ \\
\hline 9 & 2490.4 & $1591.4(-36.1 \%)$ & $1816.4(-27.1 \%)$ & $1594.8(-36.0 \%)$ \\
\hline 10 & 2490.4 & $1911.6(-23.2 \%)$ & $2041.7(-18.0 \%)$ & $1948.3(-21.8 \%)$ \\
\hline Mean & & $-30.3 \%$ & $-27.6 \%$ & $-29.2 \%$ \\
\hline
\end{tabular}
of considered scenarios. As a result, the mean TTS reduction

TABLE II

TTS $($ VEH·H) FOR DIFFERENT CONTROLLERS AND SCENARIOS 
for the 10 scenarios is also quite close the optimal one $(29.2 \%$ versus $30.3 \%$ ).

The integrated controller using MTFC and PI-ALINEA is also able to substantially improve, with respect to the nocontrol case, the behavior of the traffic system with a mean TTS reduction of $27.6 \%$ for the 10 scenarios. However, the resulting performance is slightly worse than the one obtained using the proposed controller (LB-TFC) for most of the considered scenarios. As a result, the mean TTS reduction for the 10 scenarios is slightly worse that the one obtained with LB-TFC (27.6\% versus $29.2 \%$ ).

Nevertheless, it is worth to mention that MTFC + PIALINEA shows a nice performance even taking into account that the VSLs are located downstream of the RM installations (which, to the best of our knowledge, had not been studied before in the literature).
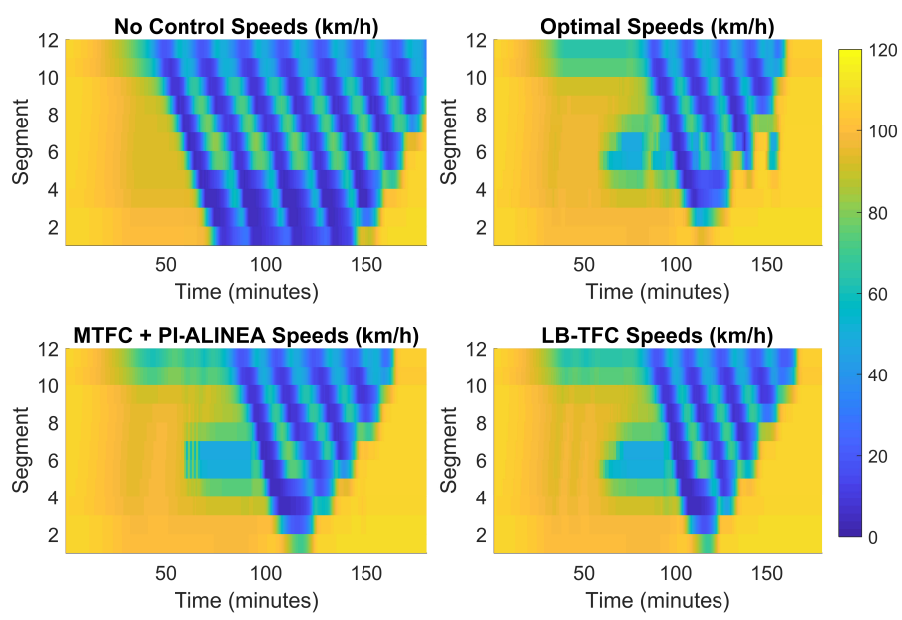

Fig. 1. Speed contour plots for Scenario 3

\section{Graphical results}

This section graphically represents the simulation results obtained for one representative scenario: Scenario 3, which uses the Mainline Demand 2 and which the same maximum queue length (200 vehicles).

Firstly, the speeds contour plots obtained for Scenario 3 using each controller are shown in Fig. 1 and the corresponding densities and outflows of the bottleneck are shown in Fig. 2.

In the figures, it can be seen that, for this scenario, it is not possible to avoid traffic breakdown and, therefore, capacity drop occurs at the bottleneck. However, the times at which the capacity is reduced are delayed compared to the no-control case (entailing a reduction in the TTS): For the no-control case the congestion is created around minute 50 while for the three simulated controllers congestion appears around minute 90 .

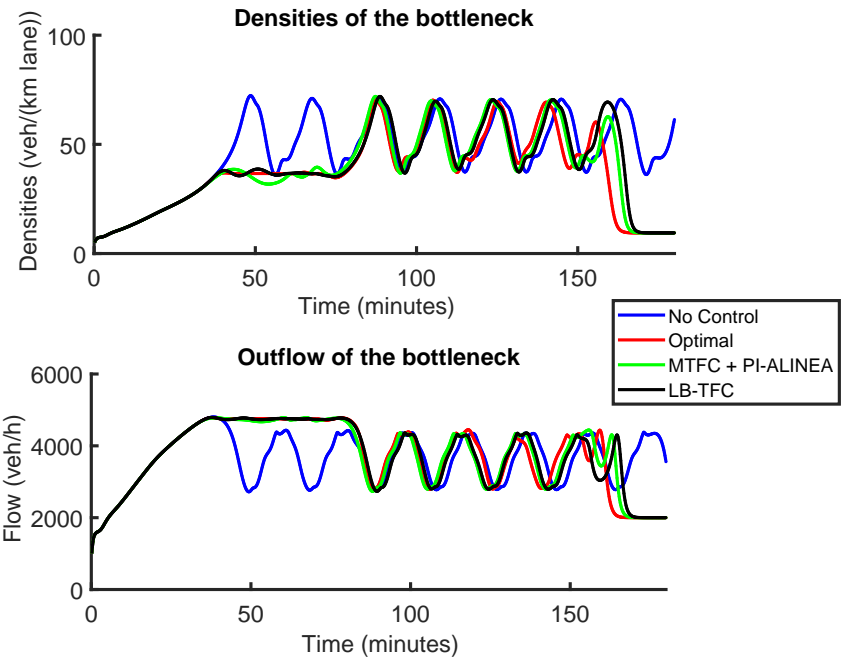

Fig. 2. Densities and outflows of the bottleneck for Scenario 3.
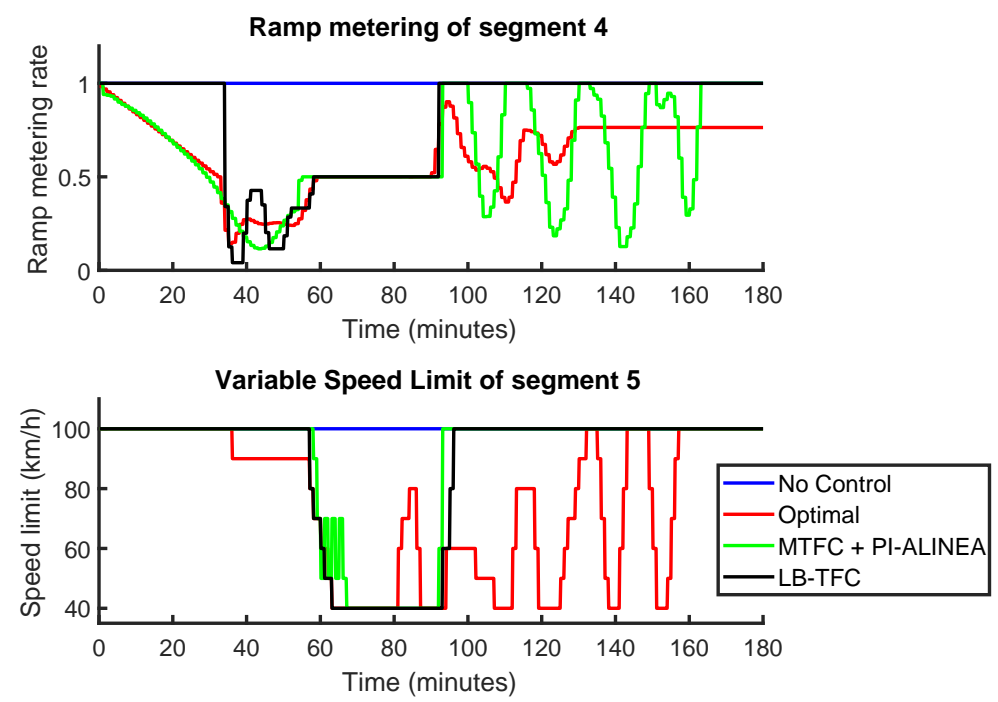

Fig. 3. RM rates, VSLs, and ramp queues for Scenario 3
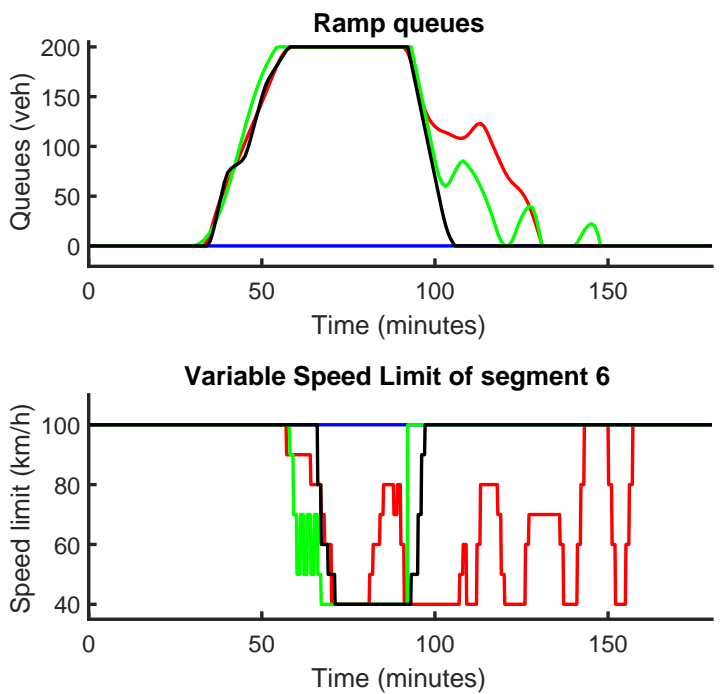
It should be noted that, as can be seen in Fig. 2, the nocontrol case is not able to recover from congestion before the end of the simulation. Although this generally undesirable, the comparison between controllers is not be affected since the rest of the simulated controllers are able to recover from congestion before the end of the simulation.

Analysing the RM rates and the speed limits, shown in Fig. 3 , it can be seen that the behavior obtained with LB-TFC and MTFC + PI-ALINEA starts to substantially differ from the optimal one when the congestion tail is created (around minute 90). However, the potential impact of the control inputs (in terms of TTS and of density and outflow of the bottleneck) is very low once the congestion queue is created (as can be seen in Fig. 2, in the numerical results of this paper and other papers [10], [11] and as has been observed by the authors in previous research).

It can also be seen in Fig. 3 that MTFC + PI-ALINEA shows oscillations for the VSL values, probably due to the existing delay (between the application of the control inputs and its effect on the bottleneck). In the case that these oscillations are undesirable, they can be avoided by changing the parameters of the controllers, which would however entail slight reductions in performance in terms of the TTS.

In fact, once the congestion tail has been created, one can argue that a safety-oriented VSL control algorithm may have more benefits than a traffic-efficiency one and that RM should just ensure that the on-ramp queue constraint is not violated.

\section{CROSS-VALIDATION}

In real applications, the values of the controller parameters will be usually computed for one case (generally, the typical demand) and applied for different scenarios. Therefore, it is important that the controllers tuned for one scenario also perform properly in other circumstances. In other words, it is necessary to have a robust controller, especially for different demand profiles.

This section analyzes the robustness, for different mainline demands and ramp queue constraints, of MTFC + PI-ALINEA and the proposed controller (by analyzing the TTS reduction for scenario $j$ when using the parameters computed for scenario $i$ ). Moreover, the optimal values of the controller parameters $\left(K_{\mathrm{I}}^{\mathrm{All}}, K_{\mathrm{I}}^{\prime}{ }^{\mathrm{All}}, K_{\mathrm{P}}^{\prime} \mathrm{All}, \bar{C}_{\mathrm{B}}^{\mathrm{All}}\right.$ and $\left.\underline{C}_{\mathrm{B}}^{\mathrm{All}}\right)$ that minimize the summation of the cost functions for the 10 scenarios have been also computed and included in the comparison (in the secondto-last column of the tables). The obtained results, shown in Table III and Table IV, support by simulation the claims about the sensitivity and robustness of LB-TFC included in [1].

More concretely, the results included in Table III show that the proposed LB-TFC controller is quite robust for different traffic conditions. In fact, for the simulated scenarios, the performance obtained with different values of $\bar{C}_{\mathrm{B}}^{\text {All }}$ and $\underline{C}_{\mathrm{B}}^{\mathrm{All}}$ is always close the one obtained with the optimal value of the

TABLE III

CROSS-VALIDATION: TTS REDUCTION WITH RESPECT TO THE NO-CONTROL CASE FOR LB-TFC WITH $\bar{C}_{\mathrm{B}}^{i}$ AND $\underline{C}_{\mathrm{B}}^{i}$ OPTIMIZED FOR SCENARIO $i$ AND With THE OPTIMAL VALUE OF THE PARAMETERS $\bar{C}_{\mathrm{B}}^{\mathrm{All}}$ AND $\underline{C_{\mathrm{B}}^{\mathrm{All}}}$

\begin{tabular}{cccccccccccccc} 
& $C_{\mathrm{B}}{ }^{1}$ & $C_{\mathrm{B}}{ }^{2}$ & $C_{\mathrm{B}}{ }^{3}$ & $C_{\mathrm{B}}{ }^{4}$ & $C_{\mathrm{B}}{ }^{5}$ & $C_{\mathrm{B}}{ }^{6}$ & $C_{\mathrm{B}}{ }^{7}$ & $C_{\mathrm{B}}{ }^{8}$ & $C_{\mathrm{B}}{ }^{9}$ & $C_{\mathrm{B}}{ }^{10}$ & $C_{\mathrm{B}}^{\mathrm{All}}$ & $\mathrm{Mean}$ \\
\hline Scen. 1 & $\mathbf{4 9 . 1} \%$ & $46.4 \%$ & $48.8 \%$ & $47.8 \%$ & $47.0 \%$ & $46.5 \%$ & $45.9 \%$ & $45.1 \%$ & $49.0 \%$ & $46.3 \%$ & $49.0 \%$ & $47.3 \%$ \\
Scen. 2 & $39.4 \%$ & $\mathbf{4 0 . 2} \%$ & $39.7 \%$ & $36.8 \%$ & $34.8 \%$ & $40.2 \%$ & $39.2 \%$ & $39.8 \%$ & $39.3 \%$ & $39.1 \%$ & $39.8 \%$ & $38.9 \%$ \\
Scen. 3 & $35.6 \%$ & $29.8 \%$ & $\mathbf{3 5 . 6} \%$ & $32.8 \%$ & $33.0 \%$ & $30.4 \%$ & $30.0 \%$ & $28.9 \%$ & $35.5 \%$ & $29.8 \%$ & $35.5 \%$ & $32.4 \%$ \\
Scen. 4 & $20.5 \%$ & $20.9 \%$ & $20.8 \%$ & $\mathbf{2 1 . 4} \%$ & $19.8 \%$ & $20.8 \%$ & $19.7 \%$ & $18.6 \%$ & $20.6 \%$ & $20.7 \%$ & $20.7 \%$ & $20.4 \%$ \\
Scen. 5 & $26.8 \%$ & $27.1 \%$ & $26.9 \%$ & $27.6 \%$ & $\mathbf{2 7 . 6} \%$ & $27.1 \%$ & $26.0 \%$ & $24.7 \%$ & $26.8 \%$ & $25.6 \%$ & $26.9 \%$ & $26.7 \%$ \\
Scen. 6 & $15.8 \%$ & $16.9 \%$ & $15.9 \%$ & $16.9 \%$ & $16.9 \%$ & $\mathbf{1 6 . 9} \%$ & $14.2 \%$ & $13.8 \%$ & $15.6 \%$ & $15.5 \%$ & $15.8 \%$ & $15.8 \%$ \\
Scen. 7 & $24.2 \%$ & $24.6 \%$ & $23.7 \%$ & $22.9 \%$ & $22.0 \%$ & $23.9 \%$ & $\mathbf{2 5 . 9} \%$ & $24.3 \%$ & $24.2 \%$ & $24.6 \%$ & $24.2 \%$ & $24.0 \%$ \\
Scen. 8 & $16.3 \%$ & $16.7 \%$ & $16.2 \%$ & $16.2 \%$ & $16.1 \%$ & $16.6 \%$ & $16.6 \%$ & $\mathbf{1 7 . 0} \%$ & $16.2 \%$ & $16.7 \%$ & $16.3 \%$ & $16.4 \%$ \\
Scen. 9 & $35.9 \%$ & $33.6 \%$ & $35.9 \%$ & $33.6 \%$ & $34.1 \%$ & $33.4 \%$ & $32.1 \%$ & $31.3 \%$ & $\mathbf{3 6 . 0} \%$ & $32.8 \%$ & $35.9 \%$ & $34.0 \%$ \\
Scen. 10 & $21.4 \%$ & $21.5 \%$ & $21.3 \%$ & $20.9 \%$ & $19.7 \%$ & $21.5 \%$ & $21.5 \%$ & $21.6 \%$ & $21.2 \%$ & $\mathbf{2 1 . 8} \%$ & $21.2 \%$ & $21.2 \%$ \\
\hline Mean & $28.5 \%$ & $27.7 \%$ & $28.5 \%$ & $27.6 \%$ & $27.1 \%$ & $27.7 \%$ & $27.1 \%$ & $26.5 \%$ & $28.4 \%$ & $27.3 \%$ & $\mathbf{2 8 . 5} \%$ & $27.7 \%$ \\
\hline
\end{tabular}

TABLE IV

CROSS-VALIDATION: TTS REDUCTION WITH RESPECT TO THE NO-CONTROL CASE FOR MTFC + PI-ALINEA WITH $K_{\mathrm{I}}^{i}, K_{\mathrm{I}}^{\prime}{ }^{\prime}$, AND $K_{\mathrm{P}}^{\prime} i$ OPTIMIZED FOR SCENARIO $i$ AND With THE Optimal VALUE OF THE PARAMETERs $K_{\mathrm{I}}^{\mathrm{All}}, K_{\mathrm{I}}^{\prime}$ All , AND $K_{\mathrm{P}}^{\prime}$ All

\begin{tabular}{|c|c|c|c|c|c|c|c|c|c|c|c|c|}
\hline & $K^{1}$ & $K^{2}$ & $K^{3}$ & $K^{4}$ & $K^{5}$ & $K^{6}$ & $K^{7}$ & $K^{8}$ & $K^{9}$ & $K^{10}$ & $K^{\mathrm{All}}$ & Mean \\
\hline Scen. 1 & $48.5 \%$ & $2.1 \%$ & $46.7 \%$ & $37.5 \%$ & $19.6 \%$ & $27.5 \%$ & $26.1 \%$ & $33.0 \%$ & $-10.2 \%$ & $11.2 \%$ & $45.9 \%$ & $24.1 \%$ \\
\hline Scen. 2 & $16.5 \%$ & $\mathbf{3 8 . 5} \%$ & $34.2 \%$ & $18.3 \%$ & $9.6 \%$ & $20.5 \%$ & $6.4 \%$ & $27.2 \%$ & $-7.9 \%$ & $-9.3 \%$ & $34.2 \%$ & $17.1 \%$ \\
\hline Scen. 3 & $-4.9 \%$ & $30.1 \%$ & $\mathbf{3 4 . 5} \%$ & $31.9 \%$ & $17.5 \%$ & $22.1 \%$ & $16.4 \%$ & $14.1 \%$ & $-6.8 \%$ & $-8.2 \%$ & $34.3 \%$ & $16.5 \%$ \\
\hline Scen. 4 & $-1.6 \%$ & $10.7 \%$ & $16.0 \%$ & $\mathbf{2 2 . 5} \%$ & $13.1 \%$ & $12.7 \%$ & $18.4 \%$ & $5.9 \%$ & $-6.9 \%$ & $-6.9 \%$ & $18.9 \%$ & $9.3 \%$ \\
\hline Scen. 5 & $-4.0 \%$ & $17.6 \%$ & $-5.0 \%$ & $-0.4 \%$ & $\mathbf{2 8 . 4} \%$ & $22.6 \%$ & $9.0 \%$ & $28.2 \%$ & $-5.5 \%$ & $-6.3 \%$ & $1.7 \%$ & $7.8 \%$ \\
\hline Scen. 6 & $-3.3 \%$ & $11.5 \%$ & $-2.1 \%$ & $-0.8 \%$ & $14.3 \%$ & $16.5 \%$ & $11.8 \%$ & $10.3 \%$ & $-6.5 \%$ & $-6.1 \%$ & $6.0 \%$ & $4.7 \%$ \\
\hline Scen. 7 & $11.5 \%$ & $-3.5 \%$ & $19.4 \%$ & $19.9 \%$ & $-4.1 \%$ & $-6.5 \%$ & $25.1 \%$ & $7.1 \%$ & $1.5 \%$ & $0.5 \%$ & $6.3 \%$ & $6.3 \%$ \\
\hline Scen. 8 & $4.0 \%$ & $-2.3 \%$ & $14.2 \%$ & $12.8 \%$ & $1.0 \%$ & $0.1 \%$ & $14.0 \%$ & $16.8 \%$ & $1.6 \%$ & $0.5 \%$ & $6.3 \%$ & $6.3 \%$ \\
\hline Scen. 9 & $-3.6 \%$ & $-7.1 \%$ & $-6.9 \%$ & $-7.0 \%$ & $-20.3 \%$ & $-14.7 \%$ & $-21.4 \%$ & $-14.8 \%$ & $27.1 \%$ & $26.4 \%$ & $-6.4 \%$ & $-4.4 \%$ \\
\hline Scen. 10 & $-14.5 \%$ & $9.1 \%$ & $-28.8 \%$ & $8.6 \%$ & $-5.9 \%$ & $-5.4 \%$ & $2.6 \%$ & $-1.0 \%$ & $16.2 \%$ & $18.0 \%$ & $4.24 \%$ & $0.3 \%$ \\
\hline Mean & $4.9 \%$ & $10.7 \%$ & $12.2 \%$ & $14.3 \%$ & $7.3 \%$ & $9.5 \%$ & $10.8 \%$ & $12.7 \%$ & $0.2 \%$ & $-0.3 \%$ & $16.0 \%$ & $8.9 \%$ \\
\hline
\end{tabular}


controller parameters. On the other hand, it can be seen in Table IV that the behavior of MTFC + PI-ALINEA is much more dependent on the value of the parameters than the one obtained with the proposed LB-TFC controller. In fact, the best mean TTS reduction for the 10 scenarios that can be obtained using MTFC + PI-ALINEA is $16.0 \%$ while using LB-TFC the best mean TTS reduction is $28.5 \%$, slightly lower than the optimal one $(30.1 \%)$. Moreover, for scenario 9, MTFC + PIALINEA significantly increases the TTS if incorrect values of the parameters are used.

It should be taken into account that Case Study I shows a considerable transport delay between the application of the control inputs and the corresponding reduction/increase of the bottleneck density. As a consequence, the performance achieved with LB-TFC is more robust (compared to MTFC+PI-ALINEA) since LB-TFC is based on a feedforward structure and MTFC+PI-ALINEA is based on a PI structure.

\section{CASE STUDY II}

\section{A. SE-30 freeway in Seville, Spain}

In the second case-study, LB-TFC has been tested and evaluated for a model of a real freeway (the SE-30 ringroad in Seville, Spain) allowing an objective evaluation of the proposed controller and a comparison with previously proposed techniques.

Fig. 4 shows a schematic representation of the considered stretch of the SE-30 freeway (from marker post 17.3 to 10.0). The modeled network includes the Centenario Bridge (segments 11 and 12 in Fig. 4), which is a bottleneck with a length of 800 meters that creates the biggest recurrent traffic jams in the region of Seville. The bridge has 2 lanes fixed in each direction and one reversible lane. The reversible lane is changed manually by the traffic operators looking at the cameras along the bridge.

For simulation purposes, it is assumed that there are 2 VSLs located on segments 9 and 10 (i.e. the two last segments before the bottleneck) and one RM installation located on segment 9 (O5). The VSLs are only allowed to take a limited number of discrete values in the set $\{20,40,60\} \mathrm{km} / \mathrm{h}$ but temporal or spatial constraints are not considered (i.e. it is allowed to reduce the value of one VSL from 60 to $20 \mathrm{~km} / \mathrm{h}$ in one time step) because the range of values allowed for the VSLs in this case study is quite small. The maximum number of the vehicles than can be waiting on the on-ramp queue is limited to 30 vehicles (otherwise, the queue of vehicles would reach an urban round-about creating an undesirable traffic jam in a crossing avenue).

\section{B. Data collection}

The data set used for this benchmark has been collected by the Traffic Control Center of Western Andalusia of the Dirección General de Tráfico (DGT), which is the government department that is responsible for the Spanish transportation network. The data set used in this work have been collected over thirteen different weekdays by 34 loop detectors. Most of the loop detectors are located in the SE-30 mainline and in

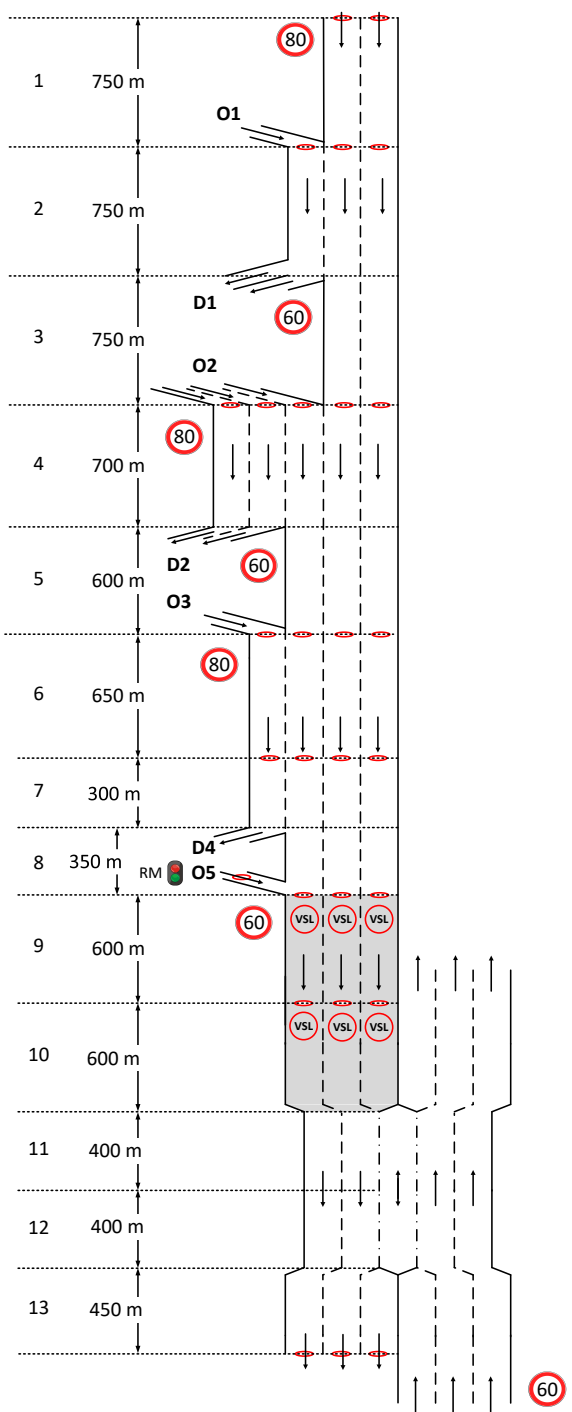

Fig. 4. Stretch of the SE-30 freeway considered for the second case study

the main on-ramps and off-ramps (marked with red circles in Fig. 4). Moreover, for some on-ramps and off-ramps without data available, we have used data from loop detectors located in the mainline of the freeways connected to SE-30. The days considered for the study are 5th, 6th, 7th, 17th, 20th, 21st, 25th, 26th, 27th and 28th of April 2017. Each loop detector provides speed and density measurements every minute. There are also data available about the state of the reversible lane on the Centenario Bridge indicating the time when the lane is closed or opened in one direction.

\section{System identification}

The freeway network described in the previous subsection has been modeled using the macroscopic second-order traffic flow model METANET [8], modified in order to include the effect of reversible lanes as proposed in [9].

The parameters of the model have been calibrated using SQP algorithms (fmincon function) and minimizing the relative quadratic error between the speeds and flows predicted 
by the model and the measurement obtained from real data such as it was done in [9] for an older data set of the SE-30 freeway.

The data set used for validation corresponds to April 28. The real data with a sample time of 1 minute have been aggregated every 10 minutes in order to reduce noise. Three different fundamental diagrams have been considered. The first one corresponds to the segments with a speed limit of $80 \mathrm{~km} / \mathrm{h}$. The second one corresponds to the segments with a speed limit of $60 \mathrm{~km} / \mathrm{h}$ and without reversible lanes. Finally, the third one corresponds to the segments with a reversible lane (i.e. the segments located on the bridge).

Both the identification parameters and the full data set are available on the website of the MSCA-IF project "Efficient Traffic Control with Variable Speed Limits: Bridging the Gap between Theory and Practical Implementation" (https://etcvsl.wordpress.com/).

\section{D. $L B-T F C$}

This sections analyzes the implementation and the tuning of LB-TFC for the SE-30 freeway.

Firstly, since LB-TFC is based on the capacity of the bottleneck and, in this case study, the capacity of the considered bottleneck is suddenly increased or decreased at any time that the reversible lane is opened or closed, the tuning parameters of LB-TFC have to be slightly modified. Therefore, due to the nature of the considered freeway (a bottleneck with a dynamically changing number of lanes), $\bar{C}_{\mathrm{B}}$ and $\underline{C}_{\mathrm{B}}$ are adjusted on-line:

$$
\begin{aligned}
& \bar{C}_{\mathrm{B}}(k)=\frac{\lambda_{\mathrm{R}}(k)}{2} \bar{C}_{\mathrm{B}} \\
& \underline{C}_{\mathrm{B}}(k)=\frac{\lambda_{\mathrm{R}}(k)}{2} \underline{C}_{\mathrm{B}}
\end{aligned}
$$

where $\lambda_{\mathrm{R}}(k)$ is the number of lanes open in the considered direction at time step $k$ (so $\lambda_{\mathrm{R}}(k)=2$ if the reversible lane is closed or open in the considered direction, and $\lambda_{\mathrm{R}}(k)=3$ if the reversible lane is open in opposite direction).

Subsequently, the control parameters of LB-TFC are identified by optimizing (1) for the calibration day (April 27, 2017). As explained in the first paper of the paper ('Part 1: Controller'), LB-TFC only needs to tune the value of two control parameters $\left(\bar{C}_{\mathrm{B}}\right.$ and $\left.\underline{C}_{\mathrm{B}}\right)$. The values obtained for the control parameters are $\bar{C}_{\mathrm{B}}=3320 \mathrm{veh} / \mathrm{h}$ and $\underline{C}_{\mathrm{B}}=2900$ $\mathrm{veh} / \mathrm{h}$. The use of robust techniques for the identification of the control parameters will be studied in future works.

\section{E. Optimal solution and MTFC + PI-ALINEA}

1) Optimal solution: The optimal solutions for the RM rates and values of VSLs have been found for each considered day. The procedure followed and the cost function used are the same that the ones presented in Section II-A. For this case study, $\psi$ and $\Omega$ have been set equal to 0.1 and $\epsilon$ has been set equal to 0.5 .
2) $M T F C+$ PI-ALINEA: Similarly as it was done for LBTFC in Section IV-D, the integrated controller MTFC + PIALINEA has to be modified in order to achieve an improved behavior in the case of a bottleneck with a changing number of lanes on the bottleneck.

This can be achieved by modifying the equation for the total desired inflow $\left(\hat{q}_{\mathrm{t}}(k)\right)$, which was proposed in [2] for bottlenecks with a fixed number of lanes. This modification is done by taking into account that at any time that the number of lanes is reduced or increased, the total desired inflow has to be reduced or increased accordingly. As a result, for the case study considered (3 lanes when the reversible lane is open and 2 lanes when the reversible lane is closed), the total desired inflow is computed using the following equation:

$$
\begin{aligned}
& \hat{q}_{\mathrm{t}}(k)= \\
& \frac{\lambda_{\mathrm{R}}(k)}{\lambda_{\mathrm{R}}(k-1)} \hat{q}_{\mathrm{t}}(k-1)+\left(\bar{K}_{P}+\bar{K}_{I}\right) \mathrm{e}_{\rho}(k)-\bar{K}_{P} \mathrm{e}_{\rho}(k-1)
\end{aligned}
$$

where $\mathrm{e}_{\rho}(k)$ is the density control error as defined in [2]. Additionally, the mainstream capacity (defined as $q_{\text {cap }}^{\mathrm{m}}$ in [2]) is adjusted on-line:

$$
q_{\text {cap }}^{\mathrm{m}}(k)=\frac{\lambda_{\mathrm{R}}}{3} q_{\text {cap }}^{\mathrm{m}}
$$

As expected, the obtained performance (in terms of TTS reduction) is better for the modified version of MTFC + PIALINEA (with respect to the original version) for both the calibration day $(9.2 \%$ using the modified version versus $6.9 \%$ for the original formulation) and the validation days.

Finally, equivalently as it was done for LB-TFC in Section IV-D, the values of the control parameters used for the simulation of MTFC + PI-ALINEA (for the entire set of days considered) have been found by minimizing the cost function (1) for April 27, 2017: $\bar{K}_{I}=5.9595 \mathrm{~km} \cdot \mathrm{lane} / \mathrm{h}, \bar{K}_{P}=202.62$ $\mathrm{km} \cdot$ lane $/ \mathrm{h}, K_{I}=0.000738 \mathrm{~h} \cdot$ lane $/ \mathrm{veh}, K_{I}^{\prime}=0.0069 \mathrm{~km} / \mathrm{h}$, and $K_{P}^{\prime}=1.161 \mathrm{~km} / \mathrm{h}$.

\section{F. Numerical results}

The numerical results for the 10 days considered are shown in Table V. For this case study, the total delay of the vehicles has been considered as an additional performance index in order to compare the results of the different controllers. The delay has been computed using the following equation:

$$
\text { Delay }=\text { TTS }-\operatorname{TTS}_{v_{i}=v_{\mathrm{f}}}
$$

where $\operatorname{TTS}_{v_{i}=v_{\mathrm{f}}}$ is the TTS that would be obtained if there were no congestion (i.e. if all the vehicles were driving at the free-flow speed of the segment where they are currently located).

In Table V, the TTS and delay obtained for each case are shown. It can be seen that the performance achieved by LBTFC is close the optimal one for the entire set of simulated days. In fact, the mean delay reduction obtained with LB-TFC is $18.0 \%$ while the optimal one is $20.6 \%$. The largest loss of performance appears for April 25, 2017 (an abnormally highly congested day) with a delay reduction of $4.3 \%$ using LB-TFC versus the optimal reduction of $10.2 \%$. 
TABLE V

TTS $(\mathrm{VEH} \cdot \mathrm{H})$ AND DELAY $(\mathrm{VEH} \cdot \mathrm{H})$ FOR DIFFERENT CONTROLLERS AND DAYS

\begin{tabular}{|c|cc|cc|cc|cc|}
\hline Day & \multicolumn{2}{|c|}{ Uncontrolled } & \multicolumn{2}{|c|}{ Optimal } & \multicolumn{2}{c|}{ MTFC } & LB-TFC \\
\hline & TTS & Delay & TTS & Delay & TTS & Delay & TTS \\
\hline April 28 & 2062.0 & 125.2 & $2048.3(-0.7 \%)$ & $111.5(-11.0 \%)$ & $2052.4(-0.5 \%)$ & $115.6(-7.7 \%)$ & $2049.5(-0.6 \%)$ & $112.6(-10.1 \%)$ \\
April 27 & 2429.9 & 496.9 & $2202.9(-9.3 \%)$ & $269.9(-45.7 \%)$ & $2206.3(-9.2 \%)$ & $273.3(-44.9 \%)$ & $2216.4(-8.8 \%)$ & $283.5(-43.0 \%)$ \\
April 26 & 2002.8 & 169.9 & $1951.2(-2.6 \%)$ & $118.6(-30.2 \%)$ & $1955.8(-2.3 \%)$ & $122.9(-27.6 \%)$ & $1952.9(-2.5 \%)$ & $120.0(-29.3 \%)$ \\
April 25 & 4149.7 & 2229.4 & $3922.3(-5.5 \%)$ & $2002.0(-10.2 \%)$ & $4213.3(+1.5 \%)$ & $2293.1(+2.9 \%)$ & $4054.3(-2.3 \%)$ & $2134.0(-4.3 \%)$ \\
April 21 & 2036.8 & 131.8 & $2015.7(-1.0 \%)$ & $110.8(-16.0 \%)$ & $2022.8(-0.7 \%)$ & $117.8(-10.6 \%)$ & $2018.7(-0.9 \%)$ & $113.7(-13.7 \%)$ \\
April 20 & 2025.6 & 172.7 & $1995.9(-1.5 \%)$ & $143.1(-17.2 \%)$ & $2014.4(-0.6 \%)$ & $161.5(-6.5 \%)$ & $2000.7(-1.2 \%)$ & $147.9(-14.4 \%)$ \\
April 17 & 1990.1 & 177.9 & $1984.0(-0.3 \%)$ & $171.8(-3.4 \%)$ & $1990.6(+0.0 \%)$ & $178.4(+0.3 \%)$ & $1984.6(-0.3 \%)$ & $172.4(-3.1 \%)$ \\
April 7 & 2179.3 & 243.8 & $2124.0(-2.5 \%)$ & $188.5(-22.7 \%)$ & $2166.1(-0.6 \%)$ & $230.5(-5.4 \%)$ & $2135.8(-2.0 \%)$ & $200.3(-17.9 \%)$ \\
April 6 & 2156.6 & 232.6 & $2094.8(-2.9 \%)$ & $170.8(-26.6 \%)$ & $2131.0(-1.2 \%)$ & $207.1(-11.0 \%)$ & $2103.3(-2.5 \%)$ & $179.3(-22.9 \%)$ \\
April 5 & 1993.1 & 173.5 & $1952.9(-2.0 \%)$ & $133.4(-23.1 \%)$ & $1964.1(-1.5 \%)$ & $144.6(-16.7 \%)$ & $1956.6(-1.8 \%)$ & $137.1(-21.0 \%)$ \\
\hline Mean & - & - & $-2,8 \%$ & $-20,6 \%$ & $-1.5 \%$ & $-12.7 \%$ & $-2.3 \%$ & $-18.0 \%$ \\
\hline
\end{tabular}

On other hand, it can be seen that MTFC + PI-ALINEA is also able to approach the optimal solution for the calibrated day, even slightly outperforming LB-TFC. However, as observed in Case Study I, the performance is substantially reduced for other days (not used for calibration). In fact, the delays and the TTS are even increased for two days (April 17 and April 25), that substantially differ from the day used for calibration. As a result, the mean TTS reduction for the 10 days period is substantially worse that the one obtained with LB-TFC and with the optimal solution ( $12.7 \%$ versus $18.9 \%$ and $20.6 \%$, respectively).

\section{G. Graphical results}

This section graphically presents the simulation results obtained for calibration day (April 27). Firstly, the speeds contour plots obtained for Scenario 1 for the uncontrolled case and for the three considered controllers (Optimal Control, MTFC + PI-ALINEA, and LB-TFC) are shown in Fig. 5:
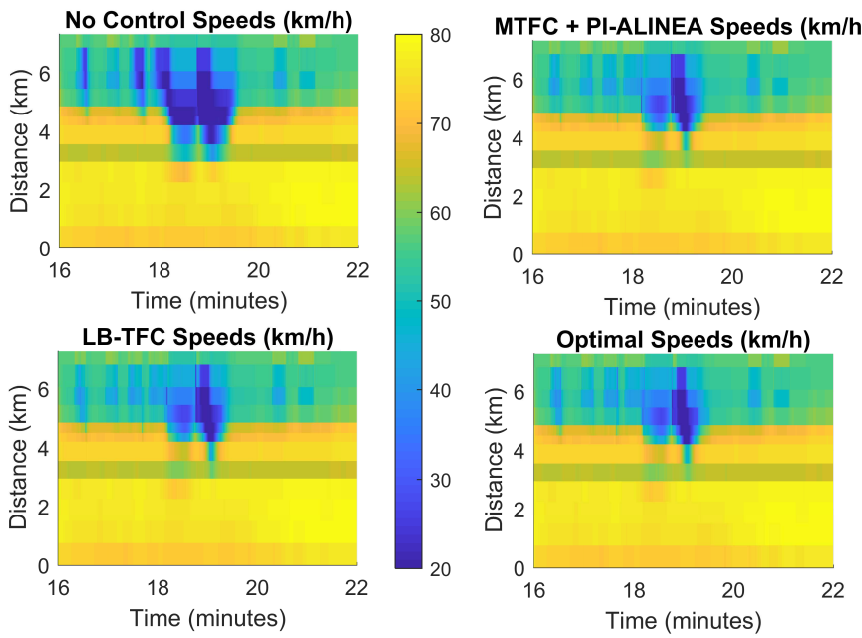

Fig. 5. Speed contour plots for April 27

In the figure, it can be seen that for the no-control case a traffic jam is created due to congestion appearing at the bottleneck, especially when the reversible lane is closed for the modeled direction. The congestion creates shock waves

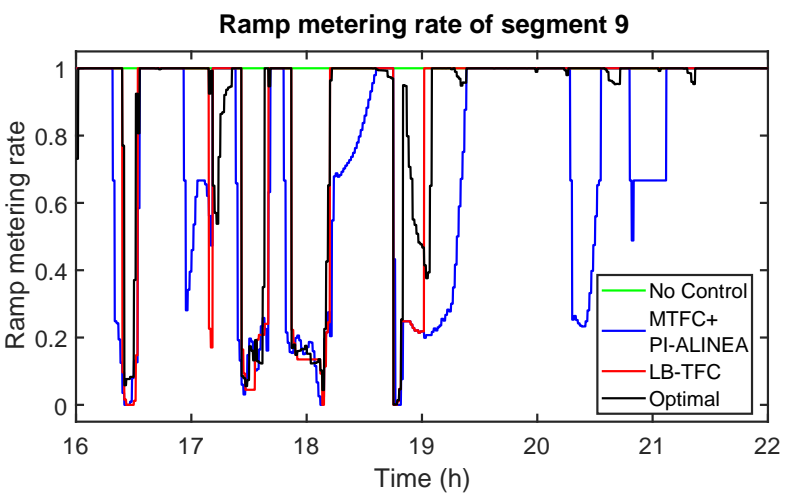

Fig. 6. Ramp metering rate for the on-ramp located on segment 9

that propagate upstream, causing a considerable decrease of the TTS as can be seen in Table V. On the other hand, the three controllers simulated are able to reduce (but not to fully resolve), the congestion created for the no-control case. This reduction of the congestion is achieved by the use of RM rates and VSLs as shown in Figures 6, 7, and 8.

In these figures, it can be seen that LB-TFC approaches the response of the optimal controller for both the RM rates and the VSL values. The integrated controller MTFC + PIALINEA is also able to approach, with a higher discrepancy than using LB-TFC, the behavior of the optimal controller.

It should be pointed out that the effects of the control inputs considered in this case study (2 VSLs and one RM installation) are limited (i.e. they are not able to fully solve the appearing traffic jam) because the speed limits only can be reduced from 60 to $20 \mathrm{~km} / \mathrm{h}$, the demands at on-ramp O5 are low, and the maximum queue allowed on the on-ramp is relatively short (30 vehicles). As a result, these control inputs are only able to completely solve relatively small traffic jams. For major traffic jams, the considered control inputs are able to reduce the delays without eliminating congestion. If more control measures were considered (such as mainstream metering, more VSLs or RM installations...) or if longer maximum values were allowed for the on-ramp queue, these major traffic jams may be fully resolved. 


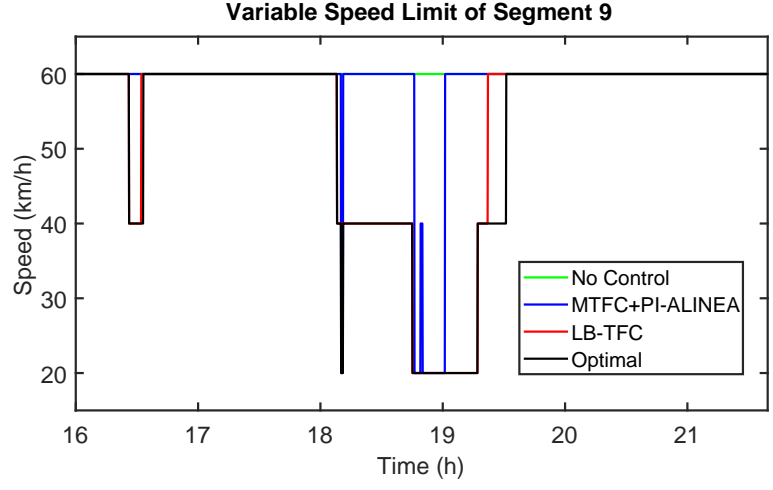

Fig. 7. VSL for segment 9

\section{TUNING}

\section{A. Tuning of the controller parameters of LB-TFC}

The response of LB-TFC can be adapted by tuning the control parameters $\bar{C}_{\mathrm{B}}$ and $\underline{C}_{\mathrm{B}}$.

The value of the control parameter $\bar{C}_{\mathrm{B}}$ should be initially set around the capacity of the bottleneck since the outflow flow $Q_{\mathrm{oB}}(k)$ will be around the capacity of the bottleneck when the density at the bottleneck is around the critical one. However, $\bar{C}_{\mathrm{B}}$ could be reduced in order to advance in time the activation of the RM installations and the VSLs. This would allow to decrease the chance of reaching the capacity drop but increasing the chance of an unnecessary activation of the $\mathrm{RM}$ installations and/or the VSLs). On the other hand, $\bar{C}_{\mathrm{B}}$ could be increased in order to delay the activation of the RM installations and the VSLs.

Equivalently, $\underline{C}_{\mathrm{B}}$ can be increased or reduced in order to delay or advance the deactivation of the the RM installations

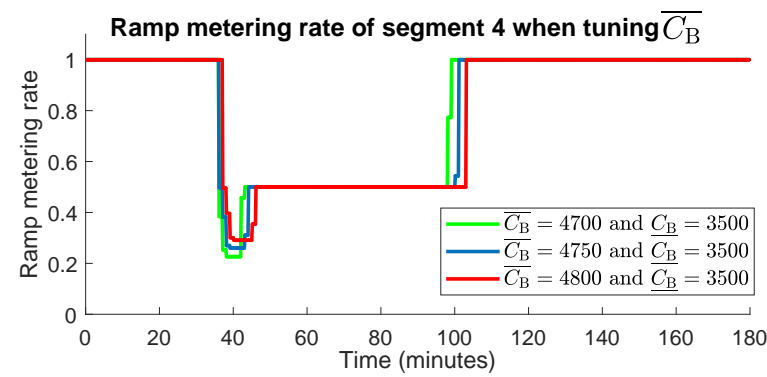

Fig. 9. Effects in the RM response when tuning the parameters of LB-TFC

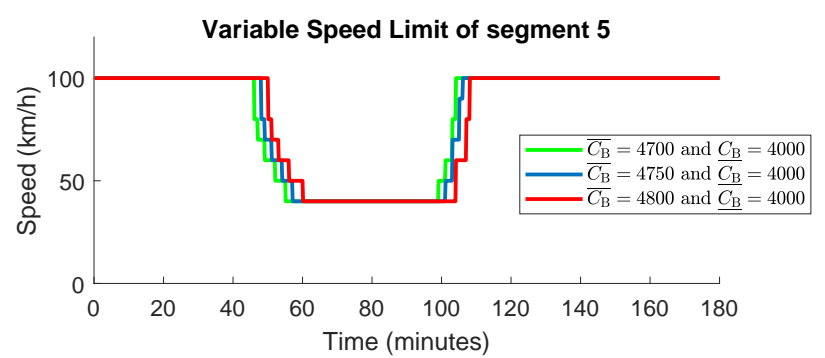

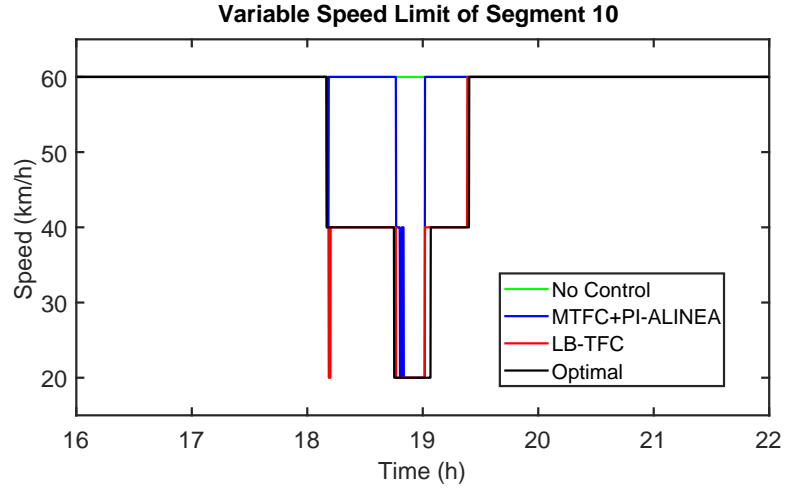

Fig. 8. VSL for segment 10

and the VSLs. The value of $\underline{C}_{\mathrm{B}}$ has to be set between the capacity of the bottleneck and the congested outflow of the bottleneck (capacity minus capacity drop). It should be taken into account the use of a value for $\underline{C}_{\mathrm{B}}$ similar to the value used for $\bar{C}_{\mathrm{B}}$ may create undesirable oscillations in the behavior of the RM rates and the VSLs. If a minimum value for the difference between $\underline{C}_{\mathrm{B}}$ and $\bar{C}_{\mathrm{B}}$ is imposed while tuning, these oscillations can be avoided.

By way of example, this section shows the differences in the behavior of the RM rate and the VSLs when the tuning parameters of LB-TFC (i.e. $\bar{C}_{\mathrm{B}}$ and $\underline{C}_{\mathrm{B}}$ ) are increased or decreased for the two used case studies.

\section{B. Tuning for Case Study I}

Firtsly, this section studies the influence of the tuning parameters $\bar{C}_{\mathrm{B}}$ and $\underline{C}_{\mathrm{B}}$ on the behavior of the proposed LBTFC controller for Case Study I. This influence is graphically
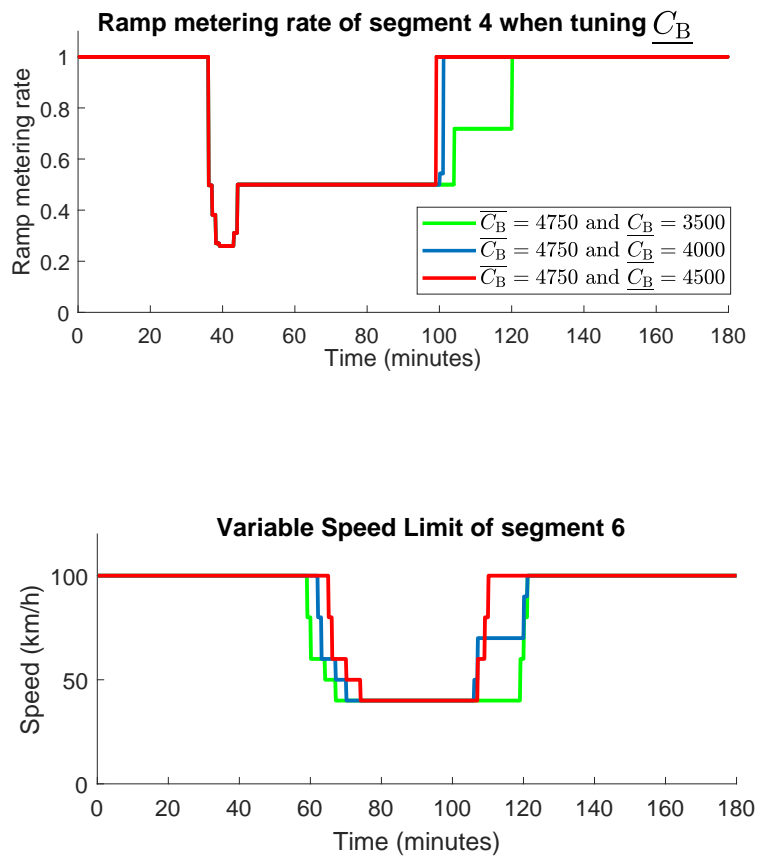

Fig. 10. Effects in the response of the VSLs of segment 5 and 6 when tuning $\bar{C}_{\mathrm{B}}$ 

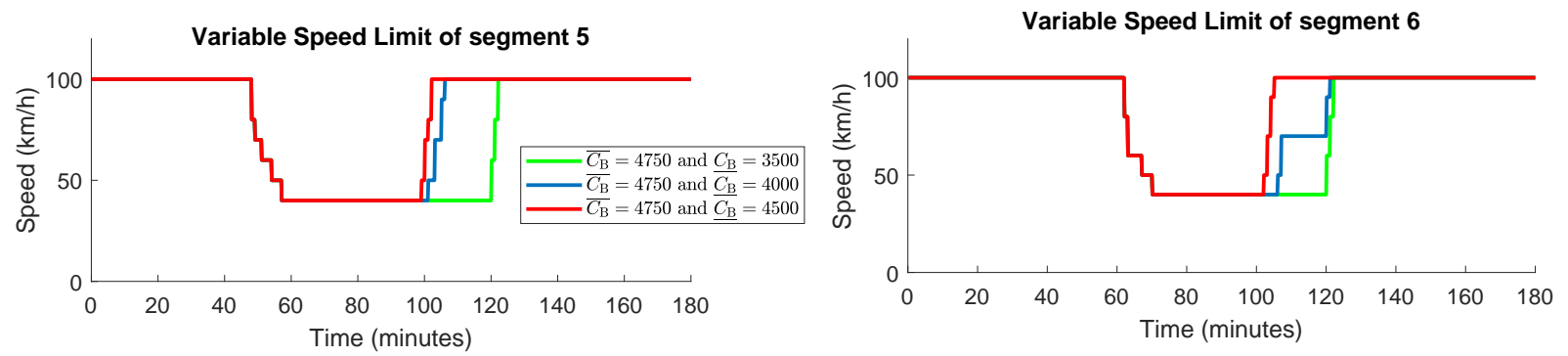

Fig. 11. Effects in the response of the VSLs of segment 5 and 6 when tuning $\underline{C}_{\mathrm{B}}$

shown in Fig. 9, 10 and 11, which displays the RM and VSL responses obtained for Scenario 2. The goal of these figures is to graphically show how the proposed controlled can be calibrated if the operator wants to delay/advance the activation/deactivation of the control inputs.

It can be seen in Fig. 9 (left plot) that the activation of RM occurs slightly sooner and is sharper for $\bar{C}_{\mathrm{B}}=4700$ veh/h than for $\bar{C}_{\mathrm{B}}=4800 \mathrm{veh} / \mathrm{h}$. Ramp metering is also deactivated sooner because the traffic jam is resolved faster but the behavior of the deactivation is not affected by $\bar{C}_{\mathrm{B}}$. Equivalently, Fig. 10 shows that the VSLs are also applied sooner for $\bar{C}_{\mathrm{B}}=4700$.

Similarly, the influence of $\underline{C}_{\mathrm{B}}$ on the response of the proposed controller is shown in Fig. 9 (right plot) and Fig. 11. As expected, it can be seen that the deactivation of the control inputs is advanced when $\underline{C}_{\mathrm{B}}$ is increased.

\section{Tuning for Case Study II}

Finally, in order to analyze how the performance of the controlled system changes with the value of the tuning parameters for Case Study II, Figure 12 shows the TTS obtained as a function of $\bar{C}_{\mathrm{B}}$ and $\underline{C}_{\mathrm{B}}$.

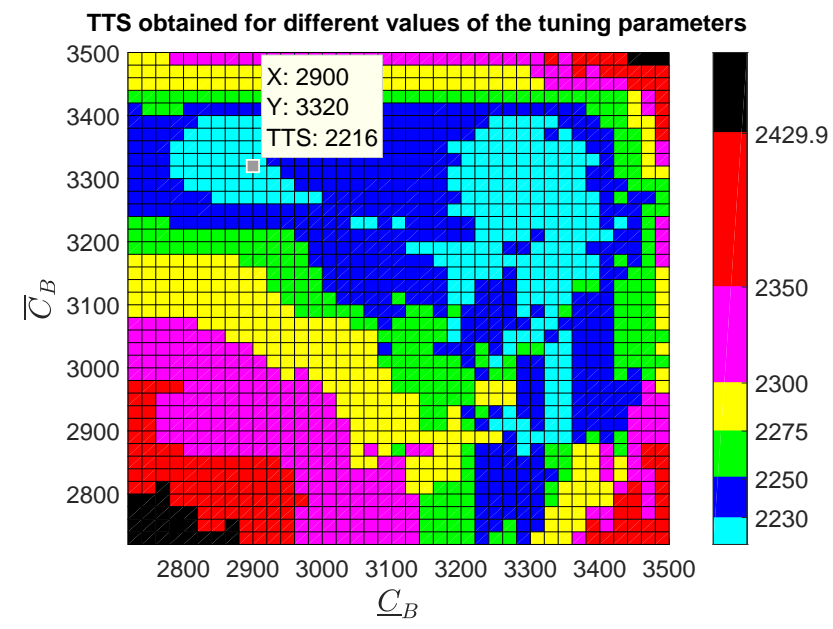

Fig. 12. Tuning for LB-TFC

In the figure, it can be seen that there are many values of $\bar{C}_{\mathrm{B}}$ and $\underline{C}_{\mathrm{B}}$ around the capacity of the bottleneck that provide a good performance (blue and cyan surfaces representing a TTS lower than $2250 \mathrm{veh} \cdot \mathrm{h}$ ). Only quite low or high values of the tuning parameters cause a performance that is worse than the one obtained for the no-control case (black surface representing a TTS higher than $2429.9 \mathrm{veh} \cdot \mathrm{h}$ ).

\section{AdVAntages AND Dis ADVANTAGES OF LB-TFC}

This section studies the main advantages and disadvantages of the proposed controller with respect to other integrated controllers for RM and VSLs. The presented advantages and disadvantages are either based on the structure of the controller or they have been observed in the two freeways simulated. Firstly, the proposed controller is compared with other easy-to-implement controllers followed by a comparison with optimization-based controllers.

\section{A. Easy-to-implement controllers}

The main advantages of the proposed controller (LB-TFC) with respect to easy-to-implement controllers for RM and VSLs proposed in literature are:

- LB-TFC is able to activate the necessary control measures before the bottleneck is congested if the flow arriving at the bottleneck is higher than the capacity of the bottleneck. Consequently, in many scenarios, this anticipation allows to avoid or reduce the congestion created at the bottleneck, substantially decreasing the TTS.

- If the capacity of the bottleneck is suddenly decreased (e.g., due to a lane closure caused by an accident) and this decrease is estimated by an incident detection system, the controller is able to activate the control measures (VSLs and/or RM installations) before the bottleneck is congested. Again, this quick activation allows to avoid or to reduce traffic jams created by incidents located at a bottleneck.

- The main advantage of LB-TFC with respect to MTFC + PI-ALINEA and other easy-to-implement integrated traffic controllers is that the obtained performance is much more robust for different demand profiles. Therefore, the proposed controller provides a very efficient performance even for scenarios that differ substantially from the one used for calibration.

- As a consequence of the inherent robustness of LB-TFC, the second main advantage of LB-TFC is that the tuning of the control parameters is much more simple than for other easy-to-implement controllers. In fact, LB-TFC can be applied in practice without a previous calibration of 
a model of the traffic network (which is necessary in most cases in order to calibrate MTFC + PI-ALINEA). Moreover, apart from the critical density, which can be estimated based on measurements and $L_{\mathrm{A}}$, which is chosen based on the network topology, there are only two parameters to calibrate $\left(\bar{C}_{\mathrm{B}}\right.$ and $\left.\underline{C}_{\mathrm{B}}\right)$ and the range of these parameters is roughly known a priori.

- LB-TFC outperforms MTFC + PI-ALINEA in terms of TTS reduction when solving the congestion created by a bottleneck (as can be seen in the results of both case studies). In fact, for the case studies considered in the second part paper, the obtained performance (in terms of TTS) is quite close to the optimal one for the entire set of simulated scenarios.

- The behavior of LB-TFC can be easily adapted to the desired one (delaying or anticipating the activation/deactivation of the control measures), just by increasing or decreasing the control parameters as previously explained in Section V.

- LB-TFC is more intuitive than PI controllers for the traffic community, which is not always familiar with automatic control, since, in contrast to previously proposed controllers such as PI-ALINEA and MTFC, the value of the control parameters have a physical meaning in the case of LB-TFC.

The main disadvantage of LB-TFC with respect to other easy-to-implement integrated algorithms is that, from an automatic control point of view, the formulation of a logic-based controller is less formal than the analysis of a PID controller, for which is easer to analyze stability and other control properties. Moreover, it has to be taken into account that LBTFC is only able to deal with congestion created a bottlenecks and that the use of at least one flow measurement located upstream of the bottleneck is necessary for the application of the controller. For shock-waves coming from downstream segments, other control algorithms should be used (such as SPECIALIST [12]). The integration of SPECIALIST and LBTFC will be a topic for a future work.

\section{B. Optimization-based controllers}

On the other hand, compared with optimization-based controllers, LB-TFC has the same advantages as other easy-toimplement controllers:

- Firstly, controller implementation is quite easy because the online computation is almost instantaneous (only a few simple equations and inequalities have to be evaluated for each control input for each sample time step). On the other hand, for an optimization-based controller the computation time needed to find an optimal solution is highly variable and it depends on the size of the network, the optimization algorithm used, the number and selection of the initial points, the horizons, etc. In addition, for large enough networks, it becomes quite difficult, if not impossible, to compute the optimal solution in real time using a second-order traffic flow model because of the exponential increase of the computation time with the size of the network.
- Moreover, easy-to-implement controllers for VSLs and RM are robust against communication and measurement failures in other segments different from the corresponding bottleneck.

\section{CONCLUSIONS}

This paper has simulated, analyzed and compared the behavior of Logic-Based Traffic Flow Control (LB-TFC), which was proposed and derivated in [1], for two case studies: a synthetic $12 \mathrm{~km}$ long freeway stretch and a stretch of SE-30 freeway in Seville, Spain).

Firstly, for the 10 scenarios considered in the first case study (a synthetic $12 \mathrm{~km}$ long freeway stretch), the simulation results have shown that the proposed controller is able to approach the optimal behavior while being quite robust for different demand profiles. Moreover, the results have also shown a significant performance increase, in terms of TTS reduction, with respect to the integrated Mainstream Traffic Flow Control (MTFC) and PI-ALINEA algorithm (MTFC + PI-ALINEA).

For the second case study, a stretch (from marker post 17.3 to 10.0) of the ring-road freeway in Seville (SE-30) has been used in order to simulate, for 10 different days, the performance of LB-TFC using real data for the afternoon peak period. The results have been also compared with the optimal solution and with MTFC + PI-ALINEA). Moreover, MTFC + PI-ALINEA has been slightly modified in order to be able to properly deal with a bottleneck with a dynamically changing number of lanes. The results show that LB-TFC provides a robust performance which, for most days, is close to the optimal one and that improves the performance obtained with MTFC + PI-ALINEA (which is even negative for two validation days).

The tuning of the control parameters of LB-TFC has also been studied for both freeways. It has been shown that the value of $\bar{C}_{\mathrm{B}}$ (or $\underline{C}_{\mathrm{B}}$ ) can be reduced in order to advance in time the activation (or the deactivation) of the control inputs or increased in order to delay the activation (or the deactivation) of the control inputs. Moreover, it has been also shown that, for the considered case studies, the range of values for the control parameters $\bar{C}_{\mathrm{B}}$ and $\underline{C}_{\mathrm{B}}$ that provide an excellent performance is quite wide.

\section{REFERENCES}

[1] J.R.D. Frejo and B. De Schutter, Logic-Based Traffic Flow Control algorithm for ramp metering and variable speed limits (Part 1: Controller), IEEE Transactions on Intelligent Transportation Systems, in press, 2020.

[2] R.C. Carlson, I. Papamichail and M. Papageorgiou, Integrated feedback ramp metering and mainstream traffic flow control on motorways using variable speed limits, Transportation Research Part C: Emerging Technologies, vol. 46, pp 209-221, 2014.

[3] A. Hegyi, B. De Schutter, and H. Hellendoorn, Model predictive control for optimal coordination of ramp metering and variable speed limits, Transportation Research Part C: Emerging Technologies, vol 13, no. 3, pp. 185-209, 2005.

[4] J.R.D. Frejo, A. Núñez, B. De Schutter, and E.F. Camacho, Hybrid model predictive control for freeway traffic using discrete speed limit signals, Transportation Research Part C: Emerging Technologies, vol. 46, pp. 309325, 2014.

[5] R.C. Carlson, I. Papamichail, M. Papageorgiou, and A. Messmer, Optimal motorway traffic flow control involving variable speed limits and ramp metering, Transportation Science, vol. 44, no. 2, pp. 238-253, 2010. 
[6] C. Pasquale, D. Anghinolfi, S. Sacone, S. Siri and M. Papageorgiou, A comparative analysis of solution algorithms for nonlinear freeway traffic control problems, IEEE 19th International Conference on Intelligent Transportation Systems (ITSC), pp. 1773-1778, Rio de Janeiro, Brazil, 2016.

[7] J.R.D. Frejo and E. F. Camacho, Global versus local MPC algorithms in freeway traffic control with ramp metering and variable speed limits, IEEE Transactions on Intelligent Transportation Systems, vol. 13, no. 4, pp. 1556-1565, 2012.

[8] M. Papageorgiou, I. Papamichail, A. Messmer and Y. Wang, Traffic simulation with METANET, Fundamentals of Traffic Simulation, Springer, 2010.

and Control, Miami Beach, FL, USA, 2018.

[9] J.R.D. Frejo, I. Papamichail, M. Papageorgiou and E.F. Camacho, Macroscopic modeling and control of reversible lanes on freeways, IEEE Transactions on Intelligent Transportation Systems, vol. 17, no. 4, pp 948-959, 2016.

[10] J.R.D. Frejo and B. De Schutter, Feed-Forward ALINEA: A ramp metering control algorithm for nearby and distant bottlenecks, IEEE Transactions on Intelligent Transportation Systems, vol. 20 (7), pp. 2448 - 2458, 2019.

[11] J.R.D. Frejo, and B. De Schutter, A logic-based speed limit control algorithm for variable speed limits to reduce traffic congestion at bottlenecks, 57th IEEE Conference on Decision and Control, pp. 198-204, 2018.

[12] A. Hegyi, S.P. Hoogendoorn, M. Schreuder, H. Stoelhorst, and F. Viti, SPECIALIST: A dynamic speed limit control algorithm based on shock wave theory, 11th International IEEE Conference on Intelligent Transportation Systems, pp. 827-832, 2008.

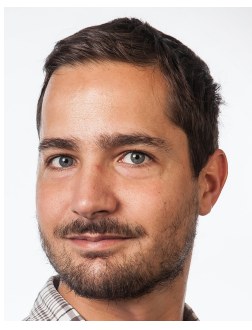

José Ramón D. Frejo received the Ph.D. degree in Automatic, Robotic and Telematics from the University of Seville in 2015 obtaining the Outstanding Doctorate Award and the the first prize of the IEEE ITS Best Dissertation Award 2016. Since February 2019 , he is working as a post-doctoral researcher for the ERC Advanced Grant OCONTSOLAR at the University of Seville, Spain. Previously, he was an MSCA-IF Research Fellow at Delft University of Technology, The Netherlands, with the Delft Center for Systems and Control (2017-2019). He was an Assistant Professor at the University of Seville (2009-2010, 2014, and 2015-2017) and at University Loyola Andalusia (2014-2015). He was a Visiting Scholar at the University of California, Berkeley (2011), at the Delft University of Technology (2012), and at the Technical University of Crete (2013 and 2017). His main research interests lie in the areas of model predictive control and freeway traffic control.

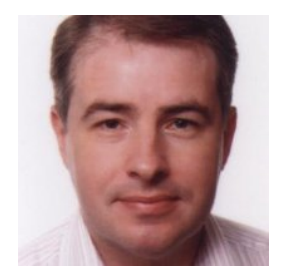

Bart De Schutter received the Ph.D. degree in applied sciences (summa cum laude with congratulations from the examination jury) from the Katholieke Universiteit Leuven, Leuven, Belgium, in 1996. Currently, he is a Full Professor and head of the department at the Delft Center for Systems and Control, Delft University of Technology, Delft, The Netherlands. He is Senior Editor for IEEE Transactions on Intelligent Transportation Systems. His current research interests include control of intelligent transportation systems, hybrid and discrete-

event systems, and multi-agent systems. 